\title{
Nature can inspire solutions for aeronautics and space sciences
}

\begin{abstract}
Life has been making R\&D activities for around four billion years engaging endless combinations with a relentless crash-test result: Reality. Nature's genius can provide a great help to solve problems. For this, engineers and biologists need to collaborate. Different methodologies can facilitate this interdisciplinary collaboration. Bringing together information from different disciplines enables understanding from different perspectives and promotes critical thinking to consider alternative solutions. As Buckminster Fuller famously observed, a system is greater than the sum of its parts. By mixing varied fields of knowledge, $1+1=3$ becomes understandable. This promises to open a new era of highly efficient innovation in aeronautics and space science.
\end{abstract}

\author{
Volume 4 Issue 3 - 2020 \\ SG Hoornaert,' $\mathrm{N}$ Lassabe, ${ }^{2} \mathrm{C}$ Finzinger, ${ }^{3} \mathrm{M}$ \\ Penalva, ${ }^{4}$ A Pechstein, ${ }^{5}$ J Chirazi, ${ }^{6}$ F Mathis ${ }^{7}$ \\ 'Morpho-Biomimicry Research \& Innovation Centre, Belgium \\ ${ }^{2}$ Cap Gemini-DEMS, France \\ ${ }^{3}$ Finzinger architecture, France \\ ${ }^{4}$ Fungus Sapiens, France \\ ${ }^{5}$ Biomimicry Academy \& phi360 Consulting, Germany \\ University of California San Diego, USA \\ ${ }^{7}$ Ecole Européenne de la Transition Ecologique (ETRE), France
}

Correspondence: SG Hoornaert, Morpho-Biomimicry Research \& Innovation Centre, Belgium, Tel +32 486477 0I5, Email biomimicry.be@gmail.com

Received: May 19, 2020 | Published: June 29, 2020

\section{Introduction}

"Human subtlety will never devise an invention more beautiful, more simple or more direct than does Nature because in her inventions, nothing is lacking and nothing is superfluous".

\section{— Leonardo daVinci}

Our solar system, with its planetary parade, including our own, appeared about 4.5 billion years ago. For us mortals, it is difficult to conceive of such long time-spans. If we project this duration onto an Earth year, it allows us to see our story differently. Consider a wellknown analogy: that our planet was born on January $1^{\text {st }}$ and we are now at December $31^{\text {st }}$ midnight. Life appears at the end of February. Multi-cellular organisms appear mid-August. The Cambrian biodiversity explosion occurs mid-November with the apparition of Fungi, Fishes, Plants and Insects. During December Amphibians, Reptiles, Mammals, Birds then Flowers appear. The first hominids walk the $31^{\text {st }}$ of December at $11 \mathrm{am}$. Homo sapiens appear at $23 \mathrm{~h} 36$. Agriculture is invented at $23 \mathrm{~h} 59$ and the industrial revolution occurs at $23 \mathrm{~h} 59$ and 58 seconds.

We are a very young species. All human history would occur during the last half hour of the last day. The industrial revolution would take place in the last 2 seconds. This perspective helps us better realize that throughout this gigantic period of time, life had the opportunity to test an almost infinite number of possibilities that have undergone evolutionary pressure, a radical crash-test: the Reality.

The beauty, power and immensity of nature are an inexhaustible source of inspiration. In mythology, there are many examples of bioinspired solutions: Daedalus and his son Icarus, Hermes, Pegasus, etc. Among the first traces of applied Bio-inspiration, Leonardo daVinci, in the $15^{\text {th }}$ century, was inspired by the flight of birds to imagine the forefathers of our planes. Bio-inspiration has sporadically led to innovations such as Velcro, which was invented by Georges de Mestral inspired by the observation he made about the burdock fruits attached to the hair of his dog. Water lily leaf veins would have inspired the
Crystal Palace, one of the largest glass and metal structures, invented by Joseph Paxton for the 1851 World Expo. For another world expo, the Eiffel tower built in 1889 was inspired by femoral bones structures. These innovations inspired by nature were mostly accidental and sporadic. Otto Herbert Schmitt, an American biophysicist and genius inventor, would have coined the term "Biomimicry" in his doctoral thesis already in 1957. In the early 1970s, two British scientists from very different disciplines began a collaboration at the University of Reading. Julian Vincent, zoologist and Georges Jeronimidis, engineer, examine the biomechanics of plants and animals. They created an interdisciplinary department to strengthen the integration between their respective disciplines: the "Center of Biomimetics" at the U/ Reading was born. However, it was not until the publication of Janine Benyus's book "Biomimicry-Innovation inspired by Nature" in 1997 that the fundamentals of biomimicry and bio-inspiration were disseminated more widely.

The volume of academic research in this area has exploded in recent years, from 100 annual articles in peer-reviewed journals in the mid-1990s to over 3000 in 2013, and this explosive trend continues. Much has been done and many methodologies have been developed to facilitate this interdisciplinary approach. Among these advancements, at the ISO level, standards have been established. The different working groups identified several themes. During these meetings, international experts from different specialties and backgrounds gathered. During daily activities, the networks of scientists around the world using a bio-inspired approach, restraints that impede the approach to be more widely employed were identified. One of the essential identified problems: interdisciplinary cooperation hardly exists. One of the reasons could be because the language used in one domain does not always correspond to the language used in other domains. There is also a major and frequent issue because of the differences of goals (the "scientific questions to be answered"). Engineers and Biologists do not necessarily have interest in the same questions. So, one challenge is to remain motivated by each other's questions. Engineers are not "wired" like biologists and have radically 
different approaches. They are, unfortunately, often unable to talk to each other and thus fail to collaborate and solve problems together. However, bridges can be established between these disciplines to bring out new, and often, revolutionary concepts. Examples of such beneficial collaborations emerge. For this to happen, some effort needs to be made to understand each other. Biologists need to acquire tools of other disciplines. For engineers, this implies understanding of some basic life's principles.

Bio-inspired innovation can help us become much more effective. At the environmental level, these innovations would enable us to improve our resilience to climate change, can regenerate our ecosystems and improve the use of natural resources. At the social level, we are witnessing the emergence of new business models, new values and new technologies. At the economic level, these innovations allow for better differentiation, improving the competitiveness of companies but also, and especially, optimization of the production processes. Circular economy and industrial ecology are beginning to be better understood even though the links with the bio-inspiration are still actually underestimated.

Regarding "return on investment", the NASA Langley research report quoted already in 2002:2 "Natural systems tend to minimize "cost" for maximum "gain" [...]. It is clear that these biological principles can also be applied to engineered systems to minimize cost and maximize gain (function). In fact, we can exploit these concepts even for deep space exploration where biological systems have yet to be discovered".

Scientific evidences show with increasing clarity that the global challenges we are facing are dramatic. ${ }^{3,4}$ In nature, we can observe all around us, the results of its unimaginable R\&D. Each living organism is like a book of knowledge, enriched over about four billion years and biologists strive to read and understand these books. Engineers, architects, designers solve problems everyday (our thanks to them) but they also need solutions for the coming challenges. They are, most of the time, unaware of the knowledge available in the huge library that is nature. Would it not be wise for engineers, architects, designers and biologists to talk to each other?

\section{Life's principles}

"Nature does nothing uselessly."

- Aristotle,

Life's principles are operating rules that "create conditions conducive to life". 5 These rules have longtime been applied unconsciously by humans, until the advent of large-scale agriculture and the industrial revolution, which then led to a rapid abandonment of these rules. Inspired by pre-established works, ${ }^{6}$ the Biomimicry 3.8 network has published a list containing all these principles of life, as shown in Figure 1.

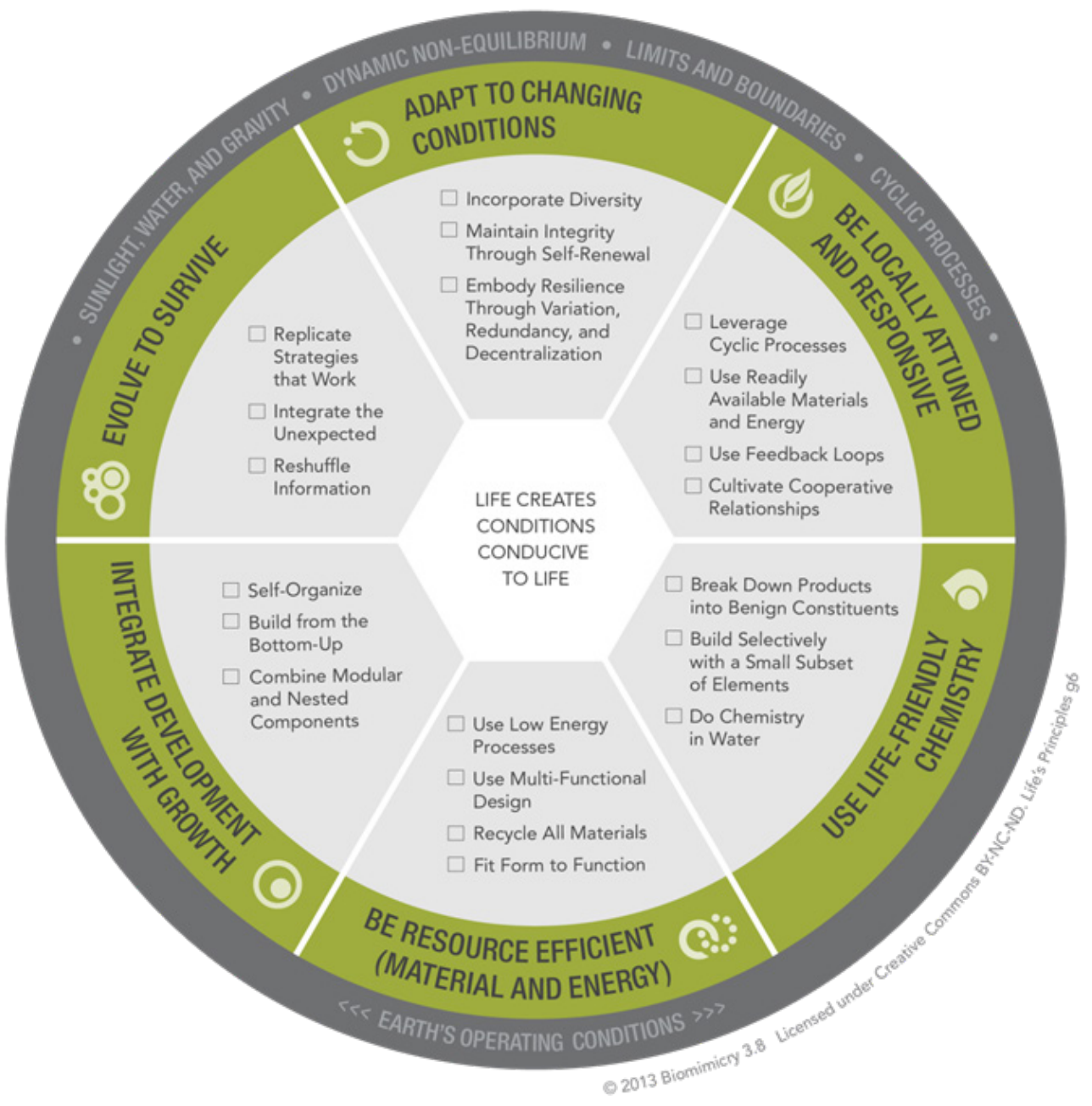

Figure I Life's principles according to the Biomimicry3.8 network. 
Life has developed these operating principles under the influence of four "Earth's operating conditions".

a. Sun, water and gravity: The sun's light has allowed life to develop by providing the necessary energy for the process of photosynthesis. Life has developed in water, an essentia molecule since the cellular processes of all living beings depend on this formidable solvent. Finally, gravity keeps us firmly rooted on the planet.

b. Non-equilibrium dynamics: The terrestrial system in which life has developed is characterized by its constant non-equilibrium nature. The conditions of the environment in which living things evolve are constantly and necessarily changing. Therefore, to deal with this impermanence, living organisms have had to develop a constant adaptability.

c. Limits and boundaries: Life has appeared in a world, which is in essence limited and finite. The only resource that is almost infinite is solar energy.

d. Cyclic processes: Many events that influence the Earth are cyclical and thus in a regular pattern (seasons, alternation between day and night, etc.). Nature operates with these cyclic processes for profit rather than trying to eliminate them.

This list of living principles is divided into six, each of which has three or four underlying sub-principles.

1. Evolve to survive: continually incorporate and embody information to ensure enduring performance. The organisms live in symbiosis with their environment while remaining autonomous. They are able to perceive and adapt to changes in their habitats. (If these behaviors are extremely complex, their study and their transposition into the field of technology open up very promising avenues for implementations: programmable materials, self-assembling robots, Internet of Things, etc.).

- Replicate strategies that work: repeat successful strategies.

- Integrate the unexpected: integrate mistakes in ways that can lead to new forms and functions.

- Reshuffle information: exchange and alter information to create new options.

2. Adapt to changing conditions: appropriately respond to dynamic contexts.

- Incorporate diversity: include multiple forms, processes, or systems to meet a functional need.

- Maintain integrity through self-renewal: persist by constantly adding energy and matter to heal and improve the system.

- Embody resilience through variation, redundancy and decentralization: maintain function following disturbance by incorporating a variety of duplicate forms, processes, or systems that are not located exclusively together (Fulfill multiple "good enough" functions instead of only one "perfect").

3. Be locally attuned and responsive: fit into and integrate with the surrounding environment. (Leaves of trees are able to collect solar energy efficiently to turn it into useful energy without rare metals importation from the other side of the world).
- Leverage cyclic processes: take advantage of phenomena that repeat themselves.

- Use readily available materials and energy: build with abundant, accessible materials while harnessing freely available energy.

Use feedback loops: engage in cyclic information flows to modify a reaction appropriately.

- Cultivate cooperation relationships: find values through winwin relationships.

4. Integrate development with growth: invest optimally in strategies that promote both development and growth. (Honeycomb structures and abalone shells are examples of hyper-resistant structures built from small amounts of abundant and soft materials.)

- Self-organize: create conditions to allow components to interact in concert to move forward an enriched system.

- Build from bottom-up: assemble components one unit at a time. These assembled elements can then have a top-down action.

Combine modular and nested components: imbricate multiple units within each other progressively, from simple to complex.

5. Use life-friendly chemistry: use chemistry that supports life processes. (Spiders are able to make stronger cables than our best Kevlar from flies, in water and at room temperature.)

- Break down products into benign constituents: use a chemistry in which decomposition result in no harmful byproducts.

- Build selectively with small subsets of elements: assemble relatively few chemical elements (mainly $\mathrm{C}, \mathrm{H}, \mathrm{O}, \mathrm{N}, \mathrm{P}$ and $\mathrm{S}$ ) in elegant ways.

- Do chemistry in water: use water as a solvent.

6. Be resource efficient: skillfully and conservatively take advantage of resource and opportunities. (Circular economy, ecological industry, are examples of this approach)

- Use low energy processes: minimize energy consumption by reducing requisite temperature, pressure, and/or time for reactions.

- Use multi-functional design: meet multiple needs with one single solution.

- Recycle all materials: keep all materials in a closed loop.

Fit form to function: select a shape or a pattern based on need.

\section{Biology vs (actual) technology}

$$
\begin{aligned}
& \text { "Men argue. Nature acts." } \\
& \text { — Voltaire }
\end{aligned}
$$

In 2006, a team of biomimetic researchers led by Julian Vincent published in the Journal of the Royal Society Interface an article entitled Biomimetics: it's practice and theory, devoted to the fundamental differences between technology and biology in problem solving. The team proposed a logical framework articulated around the following axes: to produce (need of energy and information), somewhere (space and time) and objects (substance and structure). 
They analyzed thousands of examples from biology and engineering by adapting the TRIZ method (Russian acronym for inventive problem-solving theory), a set of tools to facilitate the translation of technical solutions of one domain to another. This study highlighted significant differences in the two disciplines with the problem solving methods, quantifying them according to scale (from nanoscale to macroscale). These data were synthesized in two charts illustrating the key differences in the design, manufacture and innovation between the living world and the anthropic world. As detailed in the two diagrams in Figure 2, technology tends to solve its problems

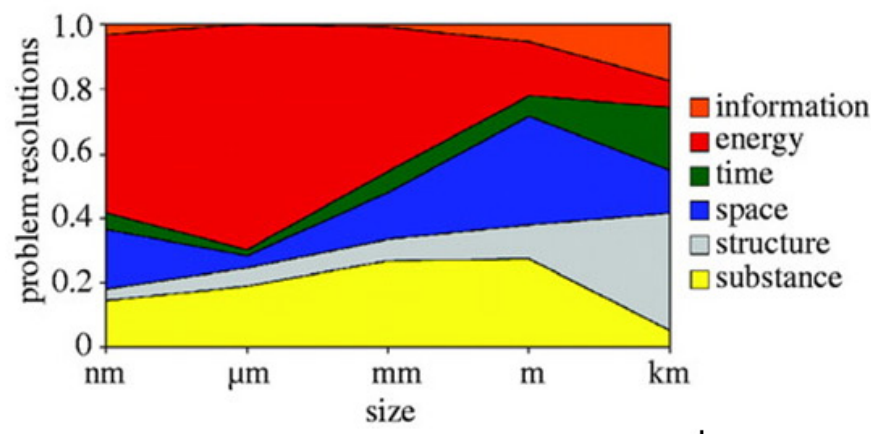

Figure 2 Comparison of resolution mode between technology (a) and living (b). ${ }^{7}$

The transfer of properties, mechanisms and principles from the field of biology to that of technologies necessarily requires reasoning by analogy. Along with materials and manufacturing, shape, surface and structure are key factors in both creative design and engineering design. It took several years to grasp the range of possibilities that these two different graphs have for design. Biomimicry sheds new light on the relationship between materials, form and function, prompting us to change the paradigm to inspire us more in the living world in the future.

To solve problems, we mainly use heat, beat and treat. Our way of solving problems is inadequate and lead us to major crises: climate changes, loss of biodiversity, desertification. These crises are such that we are witnessing the collapse of our ecosystems: the $6^{\text {th }}$ massive extinction (The last was 65 million years ago when the dinosaurs disappeared). A radical change in the way we design solutions must therefore be put in place. Could nature be our source of inspiration?

\section{Bio-inspiration}

\section{"Nature is not a place to visit. It is home."

$$
\text { — Gary Snyder }
$$

Bio-inspiration involves a radical change in the way we look at what surrounds us. It's about gaining a more humble position and seeing what we can learn from the living around us. We usually name organisms, know their characteristics, determine their places of life, ecosystem, etc. Bio-inspiration is more about seeing what we can learn from nature as if it was a mentor. And so, ask yourself the question: "What are the solutions that life has put in place throughout its evolution to solve this kind of problem?"

\section{Different level of inspiration}

Three level of inspiration have been defined: Form, process and systems. with energy reinforcement at the nanoscopic scale, matter at the millimeter scale and space at the macroscopic scale. On the other side, nature presents a much more homogeneous diagram. This increased homogeneity induces, in comparison with technology, a much greater use of structuring, temporality and information as a mode of resolution. (For example, at nanoscale level, technology uses high temperature, high pressure and many toxic components to build silicon-based microprocessors. Nature, at room temperature and in water, is able to create even thinner and subtler silicon-based structure within the Radiolaria branch).

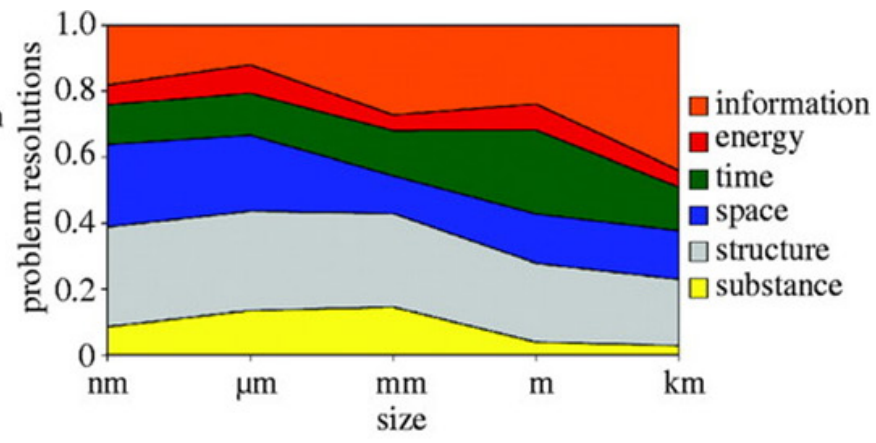

1. Form (shape, surface, texture): this is the first and simplest way to get inspiration from nature, based on the study of forms, both at macroscopic and microscopic level. Most of the industrial biomimetic applications available on the market today are at this level. This level does not necessarily imply a "sustainable" approach. (E.g.: self-cleaning surfaces - lotus leafs; Velcro - burdocks; high speed train Shinkansen500 kingfisher, owl, penguin; antifouling - sharkskin, etc.)

2. Process (and material, a series of operation): since the industrial revolution, a large majority of our materials are produced at very high temperatures (often several hundred degrees) and at high pressure, while using residual toxic solvents. Paradoxically, living organisms are able to produce at ambient temperature and pressure materials (glass, cement, etc.) whose performances are comparable (or even better) to those we produce, using a limited number of chemical elements: more than $96 \%$ of living matter consists of six basic atoms: carbon, hydrogen, oxygen, nitrogen, phosphorus and sulfur. (E.g.: energy production - photosynthesis; hyper resistant textile - spider; non-chemical adhesive - gecko; air regulation - termite mound; etc.)

3. Ecosystems (a network operating together in an ongoing (ycle): this time, the object of study is no longer the species but the relationships between species, and how they allow the ecosystem to be dynamically stable and sustainable. Subsequently, biomimetics will derive a whole series of operating rules that explain the sustainability of these ecosystems. This level does not imply disruptive technologies and so can be easily applied. It is indeed much more about organizational changes and information exchange. This third level of inspiration thus applies to higher spatial and temporal scales than the first two levels. (E.g.: Circular economy, industrial and territorial ecology are becoming more renowned 
but these approaches could be greatly improved if directly inspired by the circular functioning of ecosystems).

\section{Push or pull}

(Figure 3) In general, bio-inspiration can be conducted either according to a so-called solution-based approach (Biology push) or problem-driven (Technology pull). ${ }^{8}$ These two approaches (push or pull) present differences in their starting point and their design process characteristics. The solution-based approach describes the biomimetic development process in which knowledge of a biological system of interest is the starting point for technological design. This biological system performs a function with specific properties having a potential advantage if emulated in the technological field. It is necessary that the functioning of the biological system is analyzed in detail and finely understood so that the underlying principles responsible for the function identified can be extracted in order to deal with a problem or a technological field. Parallel to the solution-based approach, the problem-driven approach seeks to solve a practical problem, with the starting point of its process, a problem identified belonging to the technological field. New or more efficient functions are applied by identifying a biological system performing a certain function or mechanism. Then, abstract and transfer these principles to underpin the technological domain. The problem-driven approach can therefore be related to a problem-solving approach. ${ }^{9}$ It is important to understand that this approach may be badly applied. An organism is found that apparently performs the same function as the one engineers are interested in (i.e. the goal is the same). But the constraints are sometimes completely different between nature and technology. Therefore, what is optimal in nature is not necessarily optimal in the technological counterpart. Also, as mentioned above, the "objective function" that nature aims to maximize is not one-dimensional (the shark skin is not only optimized for drag reduction, but also to avoid contamination). Both engineers and biologists must be aware of the differences in constraints and goals sufficiently early in the biomimetic design process.

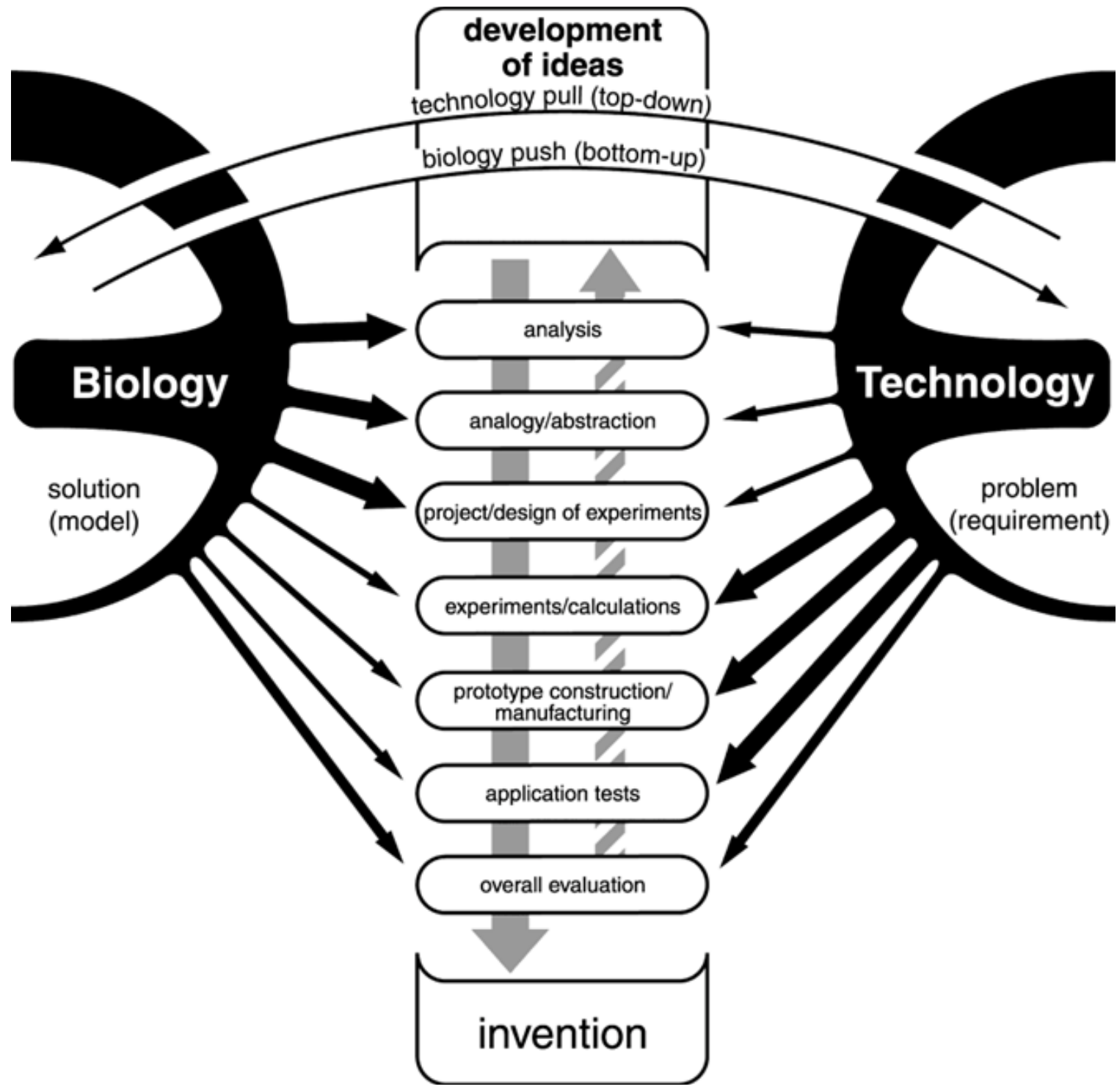

Figure 3 Schematic diagram of the development of biomimetic materials and components (push or pull). ${ }^{8}$ 
Bio-inspiration offers a unique opportunity to provide methods, guidelines and tools that rely on more than 3.8 billion years of prior problem solving through natural selection. In many areas, living organisms still outperform our technological solutions. Biomimetic solutions are interesting, not only for their ingenuity, but also for their potential for ecological resilience. Significant research has been conducted to facilitate the systematic transfer of biological knowledge to technology, to formalize methods, to generate techniques and to create tools to facilitate the biomimetic design process. ${ }^{10}$ In recent decades, biomimetic tools have been constantly developed. An inexorable increase in the tool bank available to designers interested in the bio-inspired approach is therefore to be taken into consideration. The biomimetic toolbox is divided into three main categories: the tools from the engineering sciences, the tools from the life sciences and bio-inspired design tools, having been developed with the aim of specifically facilitating the biomimetic problem-solving approach.

However, several obstacles for the implementation of the problemdriven approach are identified in the literature. ${ }^{11,12}$ Most of them are related to the interdisciplinary nature of this approach. Removing barriers to collaboration between engineer designers and biologists therefore seems to be a relevant lever to reduce the complexity of the implementation of the problem-driven biomimetic approach. An inherent element of bio-inspired approaches remains predominant, through the search for inspiration in the living: the search for biological models. Thus, regardless of the progress of biomimetic tools, integration of biologists within the biomimetic design cycles will remain an essential prerequisite. Far from being anecdotal, this prerequisite leads to a radical change in the way of thinking (Figure $4)$.

\section{Unified biomimetic model ${ }^{9}$}

As already mentioned, a large number of biomimetic processes have been developed and are still being developed today. To simplify the biomimetic approach, most of the identified process models can be combined as a model of unified problem-driven biomimetic process presented in Figure 5. This process consists of 8 steps and is not intended to be a new process model per se, but rather to be seen as an instrument for converging biomimetic process models. The process described in the model is subdivided into two phases, conceived as a symmetrical double cycle of abstraction-specification. The first phase (Step 1 to 4) focuses on the transition from technology to biology, while the second phase (Step 5 to 8) addresses the opposite approach from biology to technology. The field of knowledge considered at each stage is indicated by the color of marking, green for biology, blue for technology.

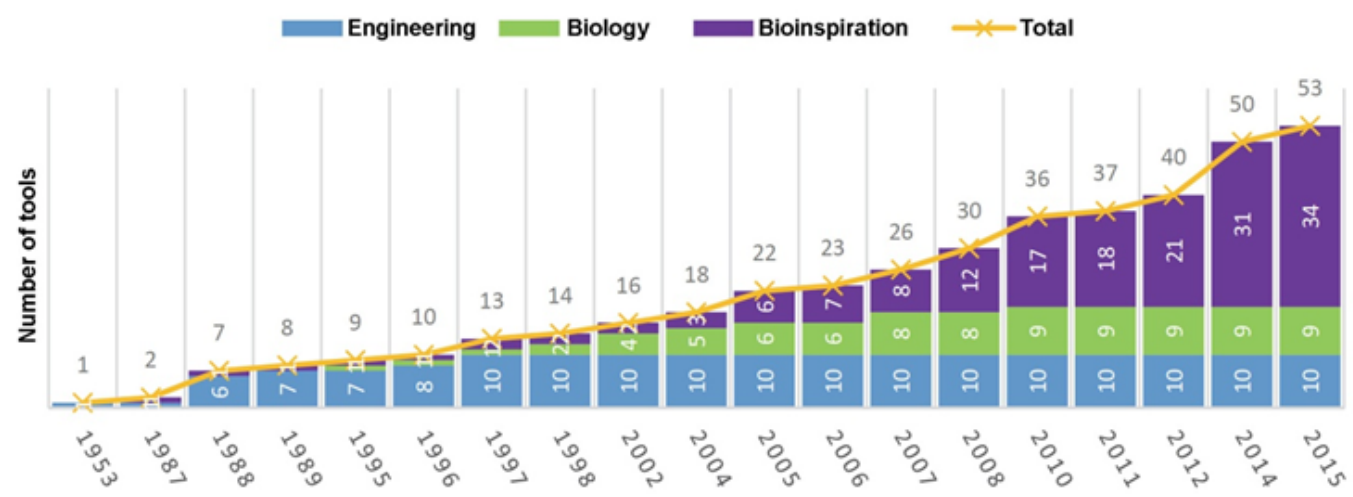

Figure 4 Appearance of biomimetic tools, classified by years. ${ }^{9}$

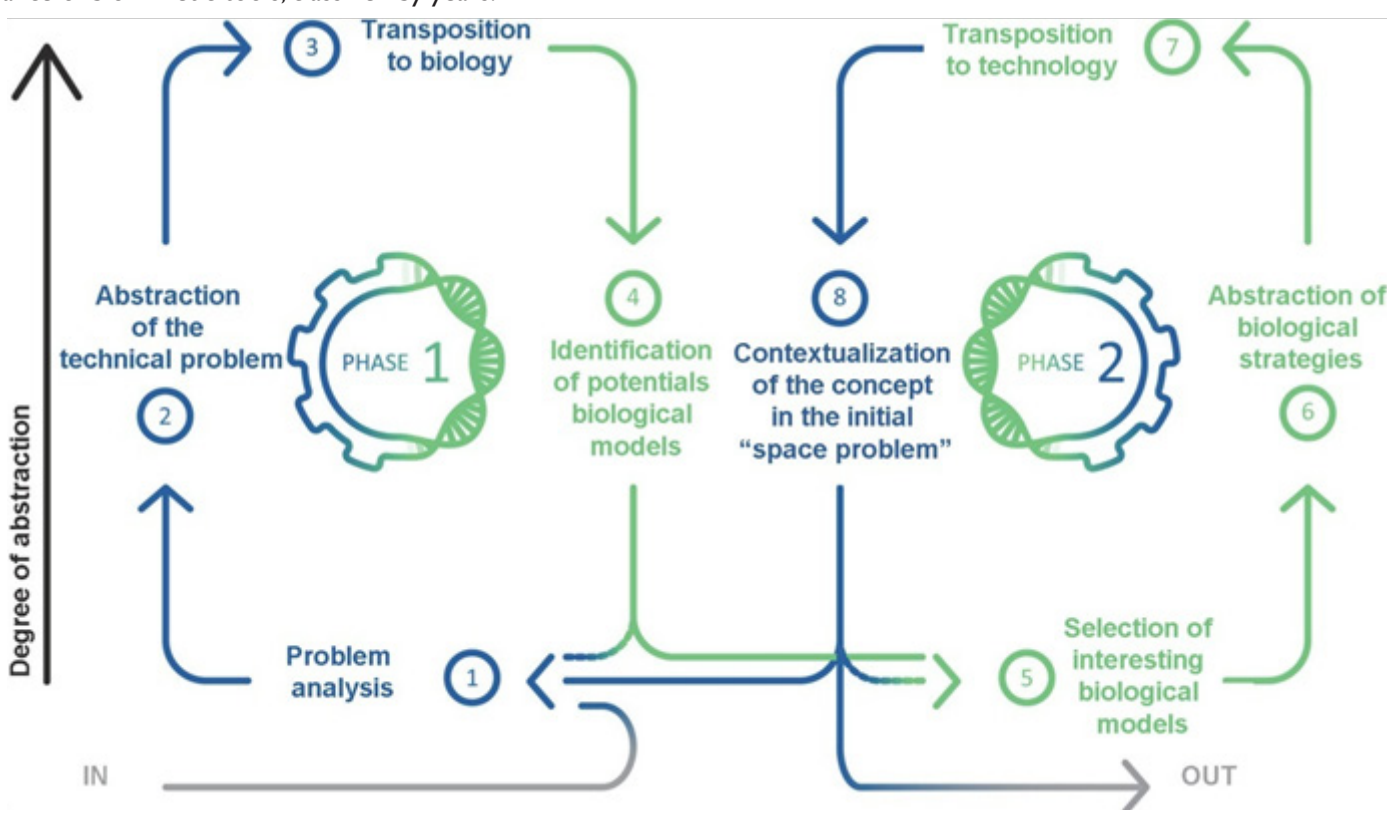

Figure 5 Unified problem-driven biomimetic process model. 
1. Problem analysis: this first step includes the assessment of the situation and/or the description of the problem. In the first scenario, a specific issue to consider has not yet been identified. The step considered then aims to identify an axis of improvement for the technical system of interest and therefore focuses on the optimization of the system. In the second scenario, one (or many) problem(s) has already been identified. It is the description of the said problem that is a key concern. The purpose of this description is to generate appropriate formalization to avoid the complications of poorly defined problems. (Using BABELE?')

2. Abstraction of the technical problem: the abstraction of the technical problem leads to the obtaining of a functional model taking into account the context as well as the constraints relating to the problem; in addition, the model clarifies the function to be achieved.

3. Transposition to biology: the generation of a generic model combined with the identification of the function(s) envisaged makes it possible to transpose the problem and its environment to biology. At this stage, a question posed to nature is usually formulated. This question is intended to allow exploration of how nature has managed to achieve one or more specific functions. This third step is important because all the results will be greatly impacted by the formulation of the query.

4. Identification of potential biological models: the transposition of the question makes it possible to identify biological models by literature research, whether using search engines or databases, or by gathering existing knowledge. Following this fourth step, a first iteration is possible. The identification of biological models can lead to a deeper understanding of the initial problem, requiring a reformulation of the problem and its biological analogy (steps 1 to 3 ).

5. Selection of the biological model(s) of interest: this selection step involves taking a step back from the upstream step. Once a pool of possibilities has been created, it is necessary to put all the occurrences identified into perspective with the initial technical problem. The purpose of this comparison is to filter biological organisms according to their relevance in order to reduce their quantity to constitute a coherent workload for the rest of the process.

6. Abstraction of biological strategies: strategies implemented by biological models must be understood and abstracted. This abstraction of biological strategies is crucial, a perfect biology-technology correspondence being, in the vast majority of cases, not feasible. Generally, abstraction leads to a functional model of the biological system.

7. Transposition to technology: the transposition of biological strategies is the next step. This step builds on the previous phase of abstraction of biological strategies and usually becomes formalized in a detailed description of the underlying principles (ex: design principles ${ }^{5}$ or functional model ${ }^{12}$ ) of the biological system under consideration, which could then be emulated technologically.

8. Contextualization of the concept in the initial problem area: once a technological emulation process has been conceptualized, the next and final step is to implement it in

${ }^{1}$ Biomimetics Analyzer of Biologically Expertised Literature for Engineers ${ }^{9}$ the initial context and evaluate it. At this point, the cycle can be successfully completed as a result of biomimetic design. If the result does not match the design expectations, the process can either be fully re-initiated or propose an iteration of the second phase by selecting one or more new interest models.

It is said that the Shinkansen500 train used the kingfisher to improve its aerodynamics and reduce noise pollution. This led to a total reduction of the noise nuisance but also of the energy needs by $10-20 \%$. The kingfisher was not the only source of innovation to solve problems related to this train. Penguins helped to improve the aerodynamics of the pantograph and especially owl feathers have inspired a way to reduce the noise nuisance of this device. Figure 6 is an illustration to better show this interdisciplinary approach model.

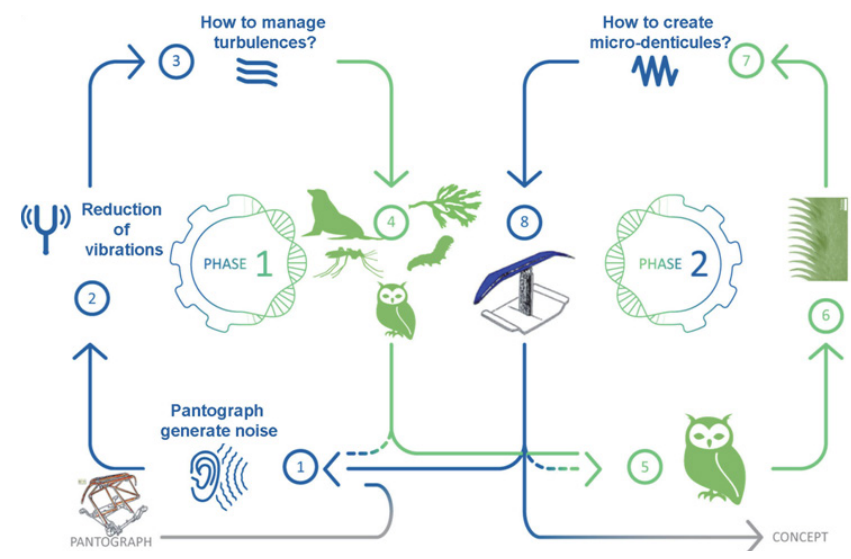

Figure 6 The unified model applied to Shinkansen500's pantograph.

The problem-driven biomimetic process appears as a double cycle. This model highlights a step often ignored: the choice of the solution or the selection of the biological model(s) of interest (step 5). This step is crucial because it is an entry point and therefore a support for the entire approach from biology to technology. The choice of an inspiration model takes into account the equivalence of biological and technological constraints and ensures the efficiency of the final product. Overall, the unified problem-driven biomimetic process model is an instrument that can help engineers and biologists collaborate as soon as the process is established. This approach allows them to create consensus on the issues and the resources required to meet the needs. In order to make the presented model even more applicable to designers, biomimetic tools can be used to facilitate the accomplishment of each of the steps described, resulting in a potential boom in biomimetics. ${ }^{9}$

Industry naturally tends to focus on engineering, but as has been demonstrated, bio-inspiration is as intrinsically linked to the life sciences. It is therefore important to be vigilant in maintaining a prominent place in biology within bio-inspired design approaches, whilst capitalizing on existing concepts and approaches within design engineering. As Buckminster Fuller famously observed, a system is greater than the sum of its part. By mixing various fields of knowledge, $1+1=3$ becomes understandable.

\section{Applications for aeronautics and space science}

"Nothing is too wonderful to be true if it be consistent with the laws of Nature."

- Michael Faraday 
NASA Glenn research center is currently developing an interesting platform named Virtual Interchange for Nature inspired Exploration (VINE). ${ }^{13}$ Many other innovative departments are already searching for nature-inspired solutions (Airbus, DARPA, MIT, etc.). The number of examples is growing. Because of a lack of space, only one application per major field of interest will be presented here that can have applications in aeronautics and space sciences. Other examples will be simply named with the related inspiration (with concrete applications between parentheses)., ${ }^{2,14-16}$

\section{Materials}

Insulation materials and mushrooms (Fungus sapiens): Neither animal nor vegetable, Fungi is a kingdom on their own still largely undiscovered. Because of their ability to degrade and transform organic matter into nutrients, fungi play a central role in the environment. This decomposition is carried out, thanks to a complex system of filaments, the mycelium consisting of hyphae that secrete specialized enzymes capable of decomposing biomass-derived polymers, such as cellulose, into monomers. The material thus formed possesses properties similar to plastic and insulating polystyrene but has the advantage of being able to be manufactured under ambient conditions, without chemicals and to be entirely compostable. In addition, these kinds of materials may have interesting irradiation insulating properties that still need to be discovered. These materials could help for Long-Term Establishment (LTE). Indeed, these organisms are creating soil and many other products, but their properties are still largely unknown or underestimated.

Other examples of materials that can be of interest for aerospace industry: pressure hyper-resistant multi-layered materials: snail, oyster, abalone (MIT); insulating materials: penguin, polar bear, mushroom; intelligent materials: pinecone; porous but thermally stable materials: sponge; etc.

\section{Surface}

Drag reduction and sharkskin (Airbus): Sharks are exceptional marine predators. They can reach top speeds of $50 \mathrm{~km} / \mathrm{h}$. They have a simple yet sophisticated method to reduce drag. Their skin is covered with denticles, tiny scales similar to teeth, whose surface is streaked with longitudinal grooves. These microgrooves modify the flow structure of water on the skin, reducing the size of vortices generated by the displacement and thereby the resistance they generate. A scientific article has demonstrated interest with a lift-to-drag ratio improvement of more than $300 \% .{ }^{17}$ And, "Airbus is considering the introduction of a sharkskin-like coating to the wings and horizontal tails of A350 XWB jetliners beginning in 2020". ${ }^{18}$ (N.B.: in addition, sharkskin limits fluid resistance while preventing microorganisms from growing).

Some other examples of surfaces that can be of interest for aerospace industry: self-cleaning surface: lotus leave (Lotusan); interferometric modulator display: butterfly (Mirasol); water harvesting: Namib desert beetle (Dar-Si-Hmad Foundation); free energy water transportation: xylem of the trees (and thorny devil lizard?); etc.

\section{Aerodynamics}

Morphing wings and birds (NASA): 19 "NASA researchers, working in concert with the Air Force Research Laboratory (AFRL) and FlexSys Inc., of Ann Arbor, Michigan, successfully completed initial flight tests of a new morphing wing technology that has the potential to save millions of dollars annually in fuel costs, reduce airframe weight and decrease aircraft noise during takeoffs and landings. The test team at NASA's Armstrong Flight Research Center in Edwards, California, flew 22 research flights during the past six months with experimental Adaptive Compliant Trailing Edge (ACTE) flight control surfaces that offer significant improvements over conventional flaps used on existing aircraft."

Other examples for aerospace applications: high-speed train: kingfisher, penguins, owl (Shinkansen500); turbine blade design: harbour seal whisker, whale and dolphins (reduce drag, + applications for new kind of airships?); ${ }^{20}$ etc.

\section{Structure}

Airless tires and honeycomb, polar bears, geckos, sea mussels, cheetah, coral, etc. (Michelin, Bridgestone, Continental, Hankook, Toyo): ${ }^{21}$ Airless tires, non-pneumatic tires or flat-free tires are tires that are not supported by air pressure. They may be used on small vehicles but also on heavy equipment. The main advantage is that they are service free, do not go flat, need to be replaced less and are much more resistant to load. Michelin has developed the Lunar wheel for NASA moon rover vehicles. ${ }^{22}$

Other examples: light and strong structure: water lily (Airbus); adherence and gecko; Light and functional hierarchical systems: Venus basket sponge (Gherkins tower in London); air regulation: termite mount (Eastgate center in Harare \& others); choc resistant materials/auxetic materials (with negative Poisson's coefficient): Oak cork, arterial cells (Zetix); Natural alveolar structure for light and resistant materials: sponges, honeybee; etc.

\section{Fabrication}

Energy production and photosynthesis: "Michael Grätzel created the field of molecular photovoltaics, being the first to conceive and realize mesoscopic photo-systems based on dyes as light harvesters that can rival and even exceed the performance of state of the art solar cells based on planar solid state $p$ - $n$ junctions. He is credited with moving the photovoltaic field beyond the principle of light absorption via diodes to the molecular level. His device presented a new paradigm since it features a 3-dimensional architecture in contrast to the planar $p-n$ junction used in conventional solar cells. The prototype of this new photovoltaic family is the dye-sensitized solar cell (DSC), also named "Grätzel cell", which employs dye molecules, pigments or quantum dots to sensitize the mesoporous oxide semiconductor scaffold. His landmark papers published in 1985 and 1991 (J.Am.Chem.Soc.1985, 107, 2988; Nature 1991, 353, 7377) had a huge impact." ${ }^{23}$

Other potential applications: highly resistant tissue and multifunctional fibers: insect \& spider fibers; low energy bacterial textile (Fungus Sapiens); radiation shields and Mycelium; breathing textiles: vegetal roots; etc.

\section{Guidance and control}

Swarm informatics and ants, barracudas, starlings (Kilobots): It's well known that ants cooperate. These tiny insects combine their efforts to accomplish complex tasks. A Mechanical Engineering team at the Georgia Institute of Technology has studied why fire ants (Solenopsis invicta) though unable individually to stay afloat, manage to float easily when in a group. ${ }^{24}$ They observed that these ants gathered to build with their own bodies ladders, chains, walls or rafts, holding each other by their mandibles and legs. These extremely 
resistant structures are also impermeable, incredibly strong and selfhealing. When ants collaborate, they seemingly become a superorganism.

Other potential applications: hybrid micro-electronics: bacteria; Informatics without brains: myxomycetes (Physarum polycephalum); sensors: black fire beetle (Melanophila acuminata); biorobotic (Toro, Cheetah-cub, Robobee); etc.

\section{System engineer}

Soft robotics and octopus (PoseiDrone): The octopus is an extremely intelligent invertebrate whose tentacles show amazing dexterity. Inspired by these features, a new type of soft robot has emerged recently. The development team has developed a new technology platform encompassing flexible mobile elements, synthetic skins lined with sensors and communication protocols to control devices in networks. PoseiDRONE consists of $76 \%$ soft elastomer and weighs $0,755 \mathrm{Kg}$. Its eight tentacles in silicone measure all $0,245 \mathrm{~m}$ and its total length is $0,78 \mathrm{~m}$. The first prototype is able to move on uneven terrain and can grab objects with its tentacles. If the performance of this first version is currently limited, it will improve the features and strategies of the next model. ${ }^{25}$

MOBOT (Multicellular soft Robot): These very interesting two last examples (swarm intelligence and soft robot) can lead to a new kind of bio-robot concept: a multicellular soft robot inspired by eukaryotic cell. To keep it short, each robot cell will work as a simplified living cell: a nucleus connected to the membrane via a cytoskeleton to manage deformation and movement (using tensegrity). Specific receptors and tools at the surface of the membrane will permit to fulfill several simple tasks. When unicellular robots will start to collaborate via special links (inspired by the natural way of cells to attach to each other), they will be able to fulfill much more complex tasks. This conformation will also theoretically be highly resistant to radiation because all electronic components will be inside a protective envelope surrounded with a liquid. This new kind of robot may have multiple applications in space but also underwater and in high radiation zones.

Other potential applications: Schmitt's rocker (Analogic-numeric conversion): calamari; artificial intelligence: brain; intelligent ramification: tree roots; robotic exoskeleton (DARPA); memory of form; self-replication; self-assembling; broken bones electrostimulation; etc.

\section{In Situ resource utilization (ISRU) and long-term establishment (LTE)}

Biosphere 2:26 This project was an experimental system built between 1987 and 1991. The purpose of this structure was to try to recreate a viable ecosystem inside a huge closed dome. One of its objectives was to evaluate the feasibility to recreate identical biospheres like earth for spatial colonization. Although this project failed, especially with regards to air recycling, this had the merit of showing the difficulty to understand and control an ecosystem. After several changes in management, the University of Arizona takes over the management of all infrastructures as a laboratory to study the effects of climate change.

Melissa's ESA:27 (Micro-Ecological LIfe Support System Alternative) is a project of the European Space Agency that aims to study autonomous systems for human food and air recycling during long-term space missions. Any long-term space mission must carry tons of consumables, which is incompatible with current logistics, hence the idea of autonomous systems to produce and recycle all these consumables. MELiSSA recovers carbon dioxide and crew waste, and forms breathable air and food from it. This is possible thanks to a set of bacteria and plants.

Uraeus0610:28 this successful collaboration between biology and architecture is an illustration that $1+1=3$. This "Science city" can be a physical and symbolical beacon of knowledge. Inspired by nature and the ancient Egyptians heritage, the project is implemented according to the Fibonacci sequence illustrated as a spiral protecting the city from the hot desiccating air of the desert. The spiral hosts a garden in its heart that spreads from the core to the main entrance following a graduation of different microclimates from tropical forest to the desert. The walls structure use local materials and is inspired by the deep hydrothermal snail Crysomallon squamiferum. Using the structure of Sphincterochila boissieri another snail, adapted to the desert, will help reduce sunshine. The inner temperature control system uses the operating principles of termite mounds (as do several buildings around the world). The Venus Flower Basket sponge (Euplectella aspergillum) inspires the tower structure, which is very light and resistant (as the Gherkin building in London). An oversized system of fog-catcher nets inspired by the Namib Desert beetle (Stenocara gracilipes) ensures sufficient water collection for the entire system. Taking inspiration from the tree xylem water evapotranspiration system and the Thorny devil lizard (Moloch horridus) ensures energy-free water transportation. Fourth generation photovoltaic panels (Graetzel's PV) will provide enough energy production. Wastewater management is using "Living machine systems" imitating the functioning of wetlands. All flows within the city are planned to operate circularly as an entire ecosystem where each waste becomes raw material. This science city project is fully bio-inspired and can potentially green deserts. The concepts developed in this project could easily be adapted for long-term human settlement on the moon and mars (Figure 7).

By 2016, European Space Agency (ESA) announced plans for building a permanent lunar base, called Moon Village. ${ }^{29}$ Practically, this very interesting concept will consist of domes built on inflatable structures and covered with regolith thanks to swarms of robots using 3D printer technology. On the moon, the days last for 15 days, as do the nights. This may pose a problem for energy supply if we use solar energy. A solution that has already been proposed to solve this would be to settle at the poles, either north or south where some places could be illuminated almost permanently, to ensure a constant energy supply. This implies to settle near or in a crater. So the other concept proposed is to stack inflatable structures in the bottom of a crater and gradually fill the rest during the stacking of these structures with regolith using 3D printer swarm robots. An elevator is even planned to pass through the stacked structures. This interesting structure will effectively protect against radiation. The problem is that putting a tube in a cone and filling the rest with regolith involves exponential (and astronomical) quantities of matter. One solution might be to use parts of the two precedent concepts and find inspiration in nature and Fibonacci: using the spiral design of Uraeus, now in 3D rather than in 2D. Like a snail shell structure set upside down. This will allow a habitat protected from radiation, drastically reduce the needs for materials and resilient due to a fully circularized environment (food, water, material). Masts will provide energy supply, even when the solar light is grazing. The construction planning will be important because zones will have different roles depending on the time. For example, the bottom of the crater will initially serve as a habitat and then as a water reservoir (where areas may need to be planned to shelter from solar storms). 


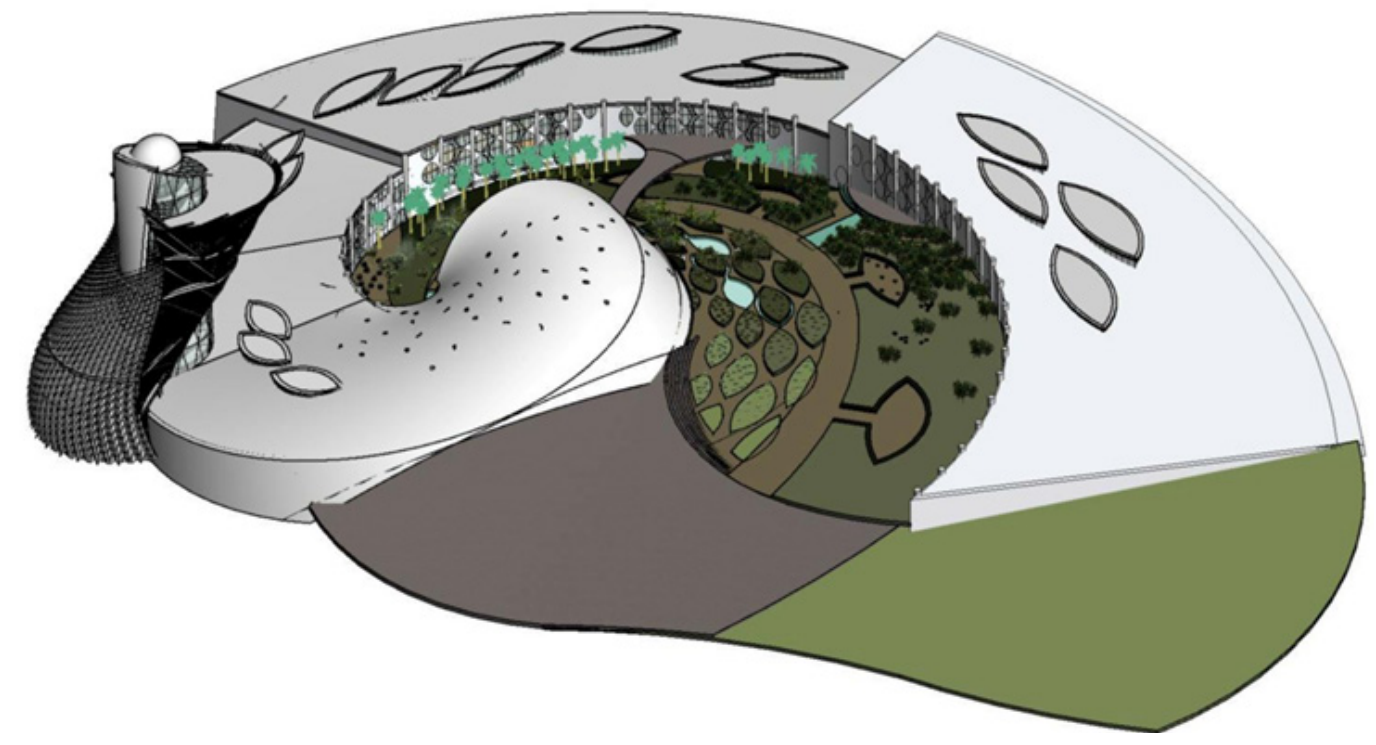

Figure 7 Illustration of science city Uraeus 0610.

Fog\&Fungi: ${ }^{30}$ As a real-life demonstration for the pertinence of the preceding project, Fog\&Fungi project used only one of the innovations proposed in Uraeus0610: FogCatchers (or CloudFishers). As already mentioned, these nets are inspired by the Namid desert beetle, a beetle living in the desert able to collect water from air using tiny structures on its carapace. These nets were installed several years ago, by the Dar-Si-Hmad Foundation on the heights of the anti-Atlas, mountain-chain southern Morocco.

The fog is captured at altitude where the size of the drops is greater. The wind pushes the droplets suspended through the mesh of the net, and they slowly descend to the gutter where they form a continuous water stream. The nets have evolved since the beginning of the project. The actual technology (CloudFishers) is the latest range of Aqualonis cloud catcher. One CloudFisher Pro consists of 4 fabrics. Total net surface: $13,5 \mathrm{~m}^{2} \times 4=54 \mathrm{~m}^{2}$. They are designed to withstand gusts of $120 \mathrm{~km} / \mathrm{h}$ and their mesh can allow a plentiful harvest approaching $55 \mathrm{~L} / \mathrm{m}^{2} /$ day in full fog days.

The harvesting of water is so efficient that there is surprisingly too much water. About $22 \mathrm{~L} / \mathrm{m}^{2}$ nets/day is collected. (Data are annualized daily averages, including days of fog and days without fog). The $1686 \mathrm{~m}^{2}$ of nets have collected about $13500 \mathrm{~m}^{3} /$ year (in 2018). The actual need for the population is $26 \mathrm{~L} /$ person/day (average year). So, the total need is about $6730 \mathrm{~m}^{3} /$ year for 142 households of 5 persons (average). This means $6670 \mathrm{~m}^{3} /$ year excess of valuable water. Due to some residual accumulation, water can only be stored for a limited period of time and needs regular flushes of $2440 \mathrm{~m}^{3}$. These flushes unfortunately induce significant soil erosion. The average estimate provides an overall understanding of the excess water originally produced for human consumption. Excess water is likely to supply an ancillary project for consumption. This is the reason why the association sought to surround itself with landscapers in addition to scientists. This surplus could be a trigger in response to many territorial issues and used to ensure many projects using biomimetic principles for soil regeneration (permaculture, agroforestry, etc.). DarSi-Hmad Foundation thus enabled collaboration with French students of National School of Landscape Architecture of Versailles-Marseille, Fungus Sapiens and the European school of Ecological Transition.
The Argan tree (Argania spinosa), a living tree in this region of the world, inspires this ongoing project. The upper part of this tree is covered with moss able to capture water (like fog-catchers). Argan trees have root a system necessarily associated with mushrooms that increase the surface area of capture, mycorhizae. These mushrooms are of crucial importance for the creation of soils. Indeed, Fungi have the ability to create soil from almost nothing thanks to specialized enzymes produced by these organisms. By creating soil, fungi (in association with bacteria) enable plants and animals to colonize land. This kind of model could be multiplied and scientifically followed to improve our understanding of eco-systemic regeneration on our planet. This will improve resilience of our cities and also greatly facilitate the long-term establishment of humanity on other planets of our solar system (as "backup").

Other interesting projects: Eden project; Sahara Forest Project; Biospheric project; Transition town network; Permaculture network; Ceinture Aliment-Terre Liege; Ecotopia; Water For All projects with Apollo 1, 2 \&3; etc.

\section{Conclusion}

"We cannot solve our problems with the same thinking we used when we created them"

\section{A. Einstein}

Access to cheap energy fuelled the industrial revolution, which has had dramatic consequences on our environment. Engineers, architects, designers, economists are solving problems everyday whilst too often being unaware that there are plenty of solutions surrounding them. With about 4 billion years of $R \& D$, nature is stacked with knowledge. Biologists, paleontologists and many others, are trying to decipher these whispered secrets. To get the best out to of this knowledge, it is important to induce a paradigm shift and establish real interdisciplinary collaborations. Engineers have much to learn from others and many tools already exist to help communication between rather diverse fields. Hopefully, examples of biomimetic solutions become increasingly numerous and international standards have even recently been created. ${ }^{31}$ 
The recent Covid-19 crisis is just a dramatic demonstration that there is an urgent need to change the way of thinking. This crisis is also an opportunity to evolve. Indeed, from a climatic point of view, unpredictable positive feedback loops seems now to be engaged, as demonstrated by the recent gigantic unpredicted fires observed during 2019. Climate is mathematically chaotic by essence. In 1977 , Ilya Prigogine got a Nobel Prize demonstrating that because of the sensitivity to initial conditions, it's almost impossible to predict long-term effect in chaotic system. The effects of global warming on Antarctica are potentially devastating. Having tipping points in mind and using simple calculation based on Archimedes' laws, global warming may lead to a quick raise of more than $1000 \mathrm{~m}$ of the Antarctica plate with all the consequences.

As briefly introduced in this article, Nature can provide many technical solutions for aeronautics and space science but also, for humankind to live in harmony with the surrounding by becoming more resilient and even multi-planetary. However, technology alone will not be enough ahead of the tremendous changes and challenges humankind is facing. Humanity, as a species, also need to become more humble and comprehend the lessons nature provides us with. The huge challenge humankind is actually facing support the ecological transition in which humanity reconcile with nature. This urgently needed paradigm shift to accelerate ecological transition is about the survival of humankind: not only to regenerate our planet but also to build "backups" away. Humanity is a very young species but adaptable and smart. It's time to shift evolve from ego to eco and start real interdisciplinary collaborations. Nature can provide solutions for aeronautics and space sciences. Please, invite biologists at the design table.

\section{Acknowledgments}

None.

\section{Conflicts of interest}

Authors declare that there is no conflict of interest.

\section{References}

1. Benyus J. Biomimicry- Innovation inspired by Nature; 1997.

2. Siochi EJ, Anders JB, Cox DE, e al. Biomimetics for NASA Langley Research Center-Year 2000 Report of Findings From a Six-Month Survey. Langley Research Center: Hampton, Virginia; 2002.

3. Steffen W, Noone K, Persson Å, e al . Planetary Boundaries: Guiding human development on a changing planet. Science. 2015;347:461.

4. Dunlop I, Spratt D. Disaster alley-Climate change conflict \& risk Breakthrough National centre for climate restoration; 2017.

5. Baumeister D, Tocke R, Dwyer J, e al. Biomimicry ressource handbook-A seedbank of best practices. CreateSpace Independent Publishing Platform; 2014.

6. Hoagland MB, Dodson B, Hauck J. Exploring the Way Life Works: The Science of Biology; 2001

7. Vincent JF, Bogatyreva OA, Bogatyrev NR, e al. Biomimetics: its practice and theory. Journal of the Royal Society Interface. 2006;3:471-482.
8. ISO/TC266. Biomimetics-Terminology, concepts and methodology. ISO $18458 ; 2015$.

9. Fayemi PE. Innovation through bio-inspired design: proposal of a model structuring biomimetic methods and formalization of a knowledge transfer tool. Ecole nationale supérieure d'arts et métiers - ENSAM; 2016.

10. Nagel JK, Nagel RL, Stone RB, e al. Function-based, biologically inspired concept generation. Artificial Intelligence for Engineering Design, Analysis and Manufacturing. 2010;24(4):521-535.

11. Yen J, Helms M, Goel A, e al. Adaptive evolution of teaching practices in biologically inspired design. In: Biologically Inspired Design. Springer; 2014. 153-199 p.

12. Helms M, Vattam SS, Goel AK. Biologically inspired design: process and products. Design studies. 2009;30(5):606-622.

13. http://www.grc.nasa.gov/vine

14. Kapseli V. Biomimetics for designers: Applying nature's processes \& materials in the real world. Thames \& Hudson Ltd; 2016.

15. Ternaux E, Gomez B, Kula B, e al. Industry of Nature: Another approach to ecology. Frame Publisher; 2012.

16. http://www.asknature.org

17. Domel AG, Saadat M, Weaver J, e al. Shark skin-inspired designs that improve aerodynamic performance. Journal of the Royal Society interface. 2018;15(139):828.

18. http://www.airbus.com/newsroom/news/en/2018/01/biomimicry-engineering-in-nature-s-style

19. NASA Successfully Tests Shape-Changing Wing for Next Generation Aviation; 2019.

20. Shyam V, Ameri A, Poinsatte P, e al. Application of Pinniped Vibrissae to Aeropropulsion. ASME Turbo Expo: Power for Land, Sea, and Air, 2A: V02AT38A023; 2015

21. Tire technology international; 2017.

22. Fazelpour M, Summers JD. Evolution of meso-structures for nonpneumatic tire development: a case study. Proceedings of the ASME 2014 International Design Engineering Technical Conferences \& Computers and Information in Engineering Conference IDETC/CIE: Buffalo, New York, USA; 2014

23. http://www.epfl.ch/labs/lpi/graetzel/

24. Phonekeo S, Mlot N, Monaenkova D, e al. Fire ants perpetually rebuild sinking towers. $R$ Soc Open Sci. 2017;4(7):170475

25. sssa.bioroboticsinstitute.it/projects/PoseiDRONE

26. biosphere2.org

27. http://www.melissafoundation.org

28. Finzinger C, Luxen J, Hoornaert SG. Uraeus0610-A Science City to green the Sahara desert; 2017.

29. http://www.esa.int/About Us/Ministerial Council 2016/Moon Village

30. Lampe T, De Saint Just C, Peñalva M, e al. Fog \& Fungi: Oasis project at the gates of the Sahara. Ecole nationale supérieure du paysage Versailles Marseille; Dar Si Hmad; Fungus Sapiens; Ecole Européenne de la Transition Ecologique (ETRE); 2018.

31. http://www.iso.org/committee/652577.html 